\title{
eJRIEPS
}

Ejournal de la recherche sur l'intervention en éducation physique et sport

$40 \mid 2017$

Varia

\section{La dynamique émotionnelle des enseignants experts en cours d'EPS : quelle relation avec la disponibilité auprès des élèves?}

Jérôme Visioli et Oriane Petiot

\section{OpenEdition}

Journals

Édition électronique

URL : http://journals.openedition.org/ejrieps/728

DOI : $10.4000 /$ ejrieps. 728

ISSN : 2105-0821

Éditeur

ELLIADD

Référence électronique

Jérôme Visioli et Oriane Petiot, « La dynamique émotionnelle des enseignants experts en cours d'EPS : quelle relation avec la disponibilité auprès des élèves ? », eJRIEPS [En ligne], 40 | 2017, mis en ligne le 01 janvier 2017, consulté le 01 août 2019. URL : http://journals.openedition.org/ejrieps/728 ; DOI :

10.4000/ejrieps.728

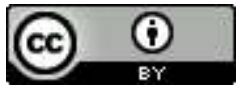

La revue eJRIEPS est mise à disposition selon les termes de la Creative Commons Attribution 4.0 International License. 


\section{eJRIEPS 40 janvier 2017}

La dynamique émotionnelle des enseignants experts en cours d'EPS : quelle relation avec la disponibilité auprès des élèves ?

Jérôme Visioli* \& Oriane Petiot**

* Université Rennes 2, France

** Université de Nantes, France

\section{Résumé}

L'objectif de cette recherche est d'analyser la dynamique émotionnelle d'enseignants experts lors de cours d'EPS. Plus précisément, il s'agit de mettre en relation cette dynamique avec la disponibilité dont les enseignants font preuve face aux élèves. En nous appuyant sur le cadre théorique et méthodologique du cours d'action (Theureau, 2006), nous avons étudié l'activité de cinq enseignants durant deux cours d'EPS, en nous centrant particulièrement sur les composantes émotionnelles de cette activité. Les résultats obtenus permettent de discuter à partir de quatre questions majeures : (1) la dynamique émotionnelle des enseignants : un plaisir d'enseigner ? ; (2) la dynamique émotionnelle des enseignants d'EPS: quelles différences entre les experts et les novices ? ; (3) entre sentiment de contrôle et prise de distance des enseignants : quels processus de régulation émotionnelle en situation ?; (4) quelles conséquences sur la formation initiale et continue des enseignants ?

Mots clés : enseignement, expertise, dynamique émotionnelle, disponibilité, cours d'action.

\section{Introduction}

Un article publié en 2014 dans la revue Movement \& Sport Sciences a proposé une analyse de l'activité d'enseignants d'EPS experts, en se focalisant sur des moments de jeu émotionnel, durant lesquels ils minimisent ou exacerbent l'expression de leurs émotions face aux élèves (Visioli, Petiot \& Ria, 2014). L'importance de l'expertise dans l'enseignement de l'EPS était alors pointée, en ce qu'elle va de pair avec des connaissances sur les manières de théâtraliser ses émotions à des fins éducatives variées. Le présent article vise à compléter les données exposées dans le précédent, en 


\section{eJRIEPS 40 janvier 2017}

se focalisant cette fois-ci sur la dynamique des émotions réellement ressenties par les enseignants, tout au long du cours, en lien avec leur disponibilité auprès des élèves.

Le rapport de la DEPP intitulé « être professeur d'éducation physique et sportive en 2009 Interrogation réalisée en mai-juin 2009 auprès de 900 professeurs d'éducation physique et sportive dans les collèges et lycées publics » (2010) souligne le mal-être de plus en plus présent chez les enseignants d'EPS, au fur et à mesure des années d'exercice. Ce constat interroge le rôle de l'expérience professionnelle dans les émotions ressenties en classe. Par ailleurs, Bui-Xuan (2011) évoque pour sa part un « plaisir créatif de l'expert » dans l'enseignement de l'EPS. Rappelons que l'expérience peut être entendue comme une des composantes de l'expertise, au même titre que la spécialisation didactique, la connaissance pédagogique, la pratique réflexive et la reconnaissance sociale (Tochon, 1993). Des recherches plus récentes insistent sur l'idée que l'expertise en enseignement, plus qu'une caractéristique stable, serait indissociable de classes de situations et de contextes particuliers (pour revue, Visioli \& Ria, 2010). Dès lors, la question se pose de savoir quelles sont les émotions ressenties par les enseignants experts lors de cours d'EPS ?

En effet, les émotions peuvent avoir une influence sur l'activité de l'enseignant face aux élèves, comme se montrer plus distant et moins attentifs à eux. Or, « le professeur est un adulte qui doit manifester sa disponibilité affective par son intérêt pour les comportements actifs ou passifs du corps de l'enseigné » (Andrieu, 2003, p. 10). La disponibilité renvoie à un esprit qui s'investit totalement dans et par l'action, dans l'ici et maintenant d'une situation (Masciotra, Roth \& Morel, 2008). Dans une enquête professionnelle ciblant l'enseignement de l'EPS, Bui-Xuan et Haye (2007) défendent l'idée que plus un enseignant développe son niveau d'expertise, plus il devient sensible aux élèves, notamment à leur plaisir. II devient alors nécessaire d'intégrer au sein des recherches les composantes émotionnelles pour analyser le plaisir et le déplaisir au travail, et questionner les manières dont les enseignants d'EPS peuvent, en contexte réel et en fonction de leur niveau d'expertise, se montrer plus disponibles aux élèves.

Les recherches s'intéressant aux émotions des enseignants sont de plus en plus nombreuses depuis les années 1980, et s'inscrivent dans des perspectives scientifiques variées, en psychologie cognitive, en psychanalyse, en action située ou dans une perspective plus sociale (voir Visioli, Petiot \& Ria, 2015, pour revue). Notamment, dans une perspective sociocognitive, Lazarus (2001) exprime l'idée que les émotions entretiennent des relations d'influence avec la cognition et l'environnement. II définit les 


\section{eJRIEPS 40 janvier 2017}

émotions comme des processus dynamiques induits par l'évaluation de la situation (« appraisal ») par rapport à sa signification pour l'individu. Les travaux s'inscrivant dans ce cadre ont étudié la régulation des émotions, c'est-à-dire le processus par lequel une personne modifie l'expérience et l'expression émotionnelle ainsi que les situations qui les suscitent pour favoriser la survenue d'émotions positives (Philippot \& Feldman, 2004). Les auteurs invitent ainsi à considérer l'importance de l'intelligence émotionnelle (ou des compétences émotionnelles) des individus, qui témoignent de leur capacité à identifier, comprendre, exprimer, réguler et utiliser leurs émotions et celles d'autrui (Mikolajczak \& Bausseron, 2013). A l'inverse, l'état émotionnel du sujet influence ces relations, les individus ayant tendance à juger les objets ou situations en accord avec la tonalité affective de leur état (Ric \& Alexopoulos, 2009).

Ramenées à l'enseignement, les recherches s'inscrivant dans ce cadre ont permis de mettre en évidence l'importance des émotions lors du face à face de l'enseignant avec ses élèves. Les premières années du métier apparaissent comme une période critique s'accompagnant d'émotions fortes et souvent négatives, en lien avec le choc de la réalité et les désillusions vis-à-vis du métier idéalisé (Sutton, 2004). Les enseignants débutants ressentent un véritable épuisement émotionnel, qui peut néanmoins être amorti par le développement de connaissances professionnelles lors des premières années d'enseignement (Dicke, Parker, Holzberger, Kunina-Habenicht, Kunter \& Leutner, 2015). Les émotions de l'enseignant influencent aussi ses propres conduites avec les élèves. Plus les enseignants sont en épuisement émotionnel, moins ils se montrent tolérants visà-vis des comportements des élèves, et moins il existe d'ordre et d'organisation dans les classes (Rascle, Bergugnat-Janot \& Hue, 2011). Par contre, l'intelligence émotionnelle de l'enseignant influence la satisfaction et le bien-être de l'enseignant en classe, ce qui a un impact sur la qualité de son travail (Mattern \& Bauer, 2014). Ces effets positifs passent, selon une étude récente, par une stabilité des émotions de l'enseignant et une certaine empathie vis-à-vis des élèves (Stojiljkovic, Stojanovic \& Doskovic, 2011). Ces résultats laissent à penser que selon les émotions ressenties par l'enseignant, son rapport aux élèves et sa manière d'être présent, attentif et finalement disponible à eux, pourront varier. Les recherches s'inscrivant dans le cadre du « cours d'action » (Theureau, 2006) adoptent quant à elles une approche des émotions en contexte. Elles y sont entendues d'une part sans catégorisation a priori (e. g., la colère), et d'autre part comme des contenus de signification non isolables des composantes de l'activité humaine. Etudier les émotions dans cette perspective peut alors apparaître paradoxal si l'on se réfère aux présupposés 


\section{eJRIEPS 40 janvier 2017}

théoriques énonçant la nécessité d'appréhender l'activité dans sa globalité, dans sa dimension insécable. Néanmoins, Ria (2006) affirme que développer un observatoire permettant d'appréhender les émotions en classe ne revient pas à isoler la dynamique émotionnelle des acteurs, mais au contraire de pouvoir étudier dans le détail les relations entre cette dynamique et d'autres composantes de l'activité humaine. Plusieurs recherches s'inscrivant dans ce cadre ont étudié les émotions des enseignants en contexte de classe, plus particulièrement en EPS, discipline considérée comme particulièrement riche en expériences émotionnelles (Durand, 2001).

Plus précisément, Ria, Sève, Durand et Bertone (2004) se sont attachés à décrire l'activité d'enseignants novices dans les situations complexes de classe, en se centrant particulièrement sur la dynamique de leurs émotions, c'est-à-dire sur les fluctuations de leurs émotions à l'échelle temporelle d'une leçon. Ils ont demandé aux enseignants d'estimer la valence et l'intensité de leurs émotions ressenties sur une échelle de « confort - inconfort » allant de -5 (inconfort) à +5 (confort) et de les commenter lors d'entretiens d'autoconfrontation (EAC). Au-delà des variations importantes de leur dynamique émotionnelle, les enseignants novices en EPS ont estimé à plus de $80 \%$ des valeurs négatives sur l'échelle d'auto-estimation des émotions. Ce constat s'explique par plusieurs éléments : (1) les enseignants novices ont des difficultés à gérer les dilemmes inhérents au métier d'enseignant ; (2) ils subissent une pression temporelle leur donnant la sensation permanente d'une course-poursuite qu'ils n'ont pas l'impression de maîtriser ; (3) ils disposent de peu de connaissances contextualisées, présentent un attachement obsessionnel au plan de leçon et craignent de se retrouver face aux élèves dans des situations inattendues. Pour toutes ces raisons, ils ressentent des émotions fréquemment intenses qui les amènent à vivre parfois moins dans l'ici et maintenant avec les élèves, et à rester centrés sur leur propre activité.

Dans la même veine, l'article publié en 2014 dans la revue Movement and Sport Sciences (Visioli et al., 2014) consiste en une adaptation du protocole de Ria et al. (2004) à des enseignants d'EPS experts. II s'est appuyé sur le constat que les travaux en sciences de l'éducation étudient peu le rôle des émotions dans l'expertise des enseignants (Visioli \& Ria, 2010), et a consisté en un recueil de données qualitatives, lors d'EAC, et quantitatives, à l'aide d'une échelle « plaisir - déplaisir » allant de -5 (déplaisir extrême) à +5 (plaisir extrême). Un tel outil a permis de mettre en évidence que les moments de jeu émotionnel s'accompagnaient d'un plaisir d'enseigner. Malgré tout, l'objet de cet article était d'analyser des moments durant lesquels les émotions étaient exacerbées, 


\section{eJRIEPS 40 janvier 2017}

théâtralisées par les enseignants, et non la dynamique de leurs émotions ressenties durant les leçons. En outre, cet article n'a pas questionné la relation entre cette dynamique et la disponibilité des enseignants auprès des élèves.

Les travaux menés dans le cadre du cours d'action s'appuient sur la conception des émotions proposée par Damasio (1999), qui distingue les concepts d'états affectifs, d'émotions et de sentiments. Les états affectifs sont des modifications neurophysiologiques de l'état de l'organisme constituant un processus continu d'adaptation de l'acteur à son environnement. Ils sont moins des états statiques que des processus dynamiques constamment modifiés. Dans cette perspective, les émotions sont définies comme des moments saillants, ponctuels et nettement isolables de la dynamique continuelle de ces états affectifs. Les sentiments, ou émotions significatives, sont quant à eux de l'ordre de la prise de conscience des émotions. Finalement, Ria et al. (2004) ont ouvert une piste de recherche mettant en avant et interrogeant les relations entre émotions, cognitions et actions au sein de l'activité humaine. D'ailleurs, Theureau (2004) reconnaît que dans les premiers développements du cours d'action, les émotions n'étaient pas une composante de l'expérience particulièrement étudiée. Selon l'auteur, « obéissant au principe de n'exposer de la théorie que ce qui en avait été effectivement mis en œuvre dans la conception ergonomique jusqu'à cette date, nous avions laissé de côté quelques éléments parmi les plus originaux de cette démarche : la considération des sentiments (ou émotions significatives pour l'acteur) 》 (p. 4).

Dans le cadre de cette recherche, nous souhaitons nous inscrire en continuité avec les travaux déjà réalisés dans le cadre du cours d'action, et étudier la dynamique de la valence et de l'intensité des émotions significatives d'enseignants d'EPS experts, c'est à dire le flux de leurs émotions lors de cours d'EPS. En outre, il s'agit de questionner la relation entre la dynamique émotionnelle et la disponibilité dont les enseignants font preuve dans l'interaction avec les élèves.

\section{Cadrage théorique}

Cette recherche s'inscrit dans le cadre théorique du cours d'action (Theureau, 2006) pour trois raisons principales. Premièrement, ce cadre s'inscrit dans une conception « énactive » de l'activité humaine (Varela, 1989), qui considère l'activité comme émergeant d'un couplage structurel asymétrique entre l'individu et l'environnement, en ce sens que la cognition n'existe que dans l'interaction entre l'acteur et le monde qui l'entoure, à travers les significations locales qu'il attribue à la situation. Autrement dit, les 


\section{eJRIEPS 40 janvier 2017}

émotions sont entendues comme des contenus de signification et sont éprouvées en lien avec les propres perceptions de l'acteur dans la situation (Colombetti \& Thompson, 2008). Plus généralement, le paradigme de l'énaction a des implications importantes en ce qui concerne la manière de considérer les émotions, en ce sens qu'elles sont sans cesse codéterminées par le couplage entre l'acteur et l'environnement (Colombetti \& Thompson, 2008). A ce titre, la valence et l'intensité des émotions ressenties par l'enseignant en classe sont davantage le fruit du couplage enseignant-environnement que le résultat de stratégies prédéterminées et d'une régulation interne.

Deuxièmement, ce cadre suppose une conception située de l'activité humaine consistant à l'ancrer dans le contexte dans lequel elle émerge (Lave, 1988). Etudier les émotions dans cette perspective nécessite d'intégrer les contextes, notamment sociaux, culturels, spatiaux et temporels dans lesquels elles émergent (Barbier \& Galatanu, 1998). D'un point de vue temporel, l'intensité et la valence des émotions ressenties par un acteur évoluent à mesure de son cours d'action et devraient ainsi être entendues dans leur dynamique (Damasio, 1999). Ce contexte est aussi social : le niveau individuel et le niveau social de l'activité sont entendus comme mutuellement constitutifs (Sève, Theureau, Saury \& Haradji, 2009). Par ailleurs, ce contexte est culturel: les sports ont une essence émotionnelle liée à leurs significations culturelles et symboliques. En EPS, les APSA sont potentiellement porteuses d'émotions particulières. II est donc essentiel d'envisager les émotions de l'enseignant en relation étroite avec le contexte de classe, notamment social, culturel et temporel.

Troisièmement, ce cadre opte pour une conception complexe et singulière de l'activité humaine et des émotions. D’après Varela (1999), les différentes composantes de l'activité, qu'elles soient émotionnelles, cognitives ou corporelles, sont continuellement imbriquées et indissociables. Dans le cadre du cours d'action, Theureau (2006) propose d'étudier ces composantes indissociables en reconstruisant le cours d'expérience des acteurs, c'est-àdire leur construction de sens tout au long de leur activité. Selon lui, ces composantes ont trait aux préoccupations, aux attentes, aux perceptions ou encore aux connaissances de l'acteur en situation. De plus, chaque activité est à la fois singulière dans la mesure où elle ne se reproduit jamais à l'identique, mais présente toujours des traits communs, des traits de généralité ou de typicalité avec d'autres activités (Rosch, 1978). Leur mise en évidence pourra alors nous permettre de comprendre comment l'enseignant, en lien avec ses émotions ressenties, prend en compte les élèves et finalement, se montre disponible auprès d'eux. 


\section{eJRIEPS 40 janvier 2017}

En synthèse, l'objectif de notre recherche est : (1) d'analyser le cours d'expérience d'enseignants d'EPS experts et particulièrement la dynamique de leurs émotions ressenties lors du cours ; (2) de mettre en évidence le rôle de l'expertise des enseignants dans leur dynamique émotionnelle tout au long du cours d'EPS, notamment à partir de leurs connaissances mobilisées; (3) de questionner la dynamique émotionnelle des enseignants experts durant le cours d'EPS, en lien avec la manière dont ils se montrent disponibles auprès des élèves.

\section{Méthode}

Les étapes méthodologiques exposées dans cette section sont en concordance avec celles décrites dans l'article publié précédemment (Visioli et al., 2014), notamment en ce qui concerne la sélection des participants et des situations étudiées, mais aussi le recueil de données. Elles s'en distinguent particulièrement au niveau du traitement des données, que nous spécifions au regard de l'objet de cet article.

3. 1. Participants et situations étudiées

Nous avons utilisé les propositions de Tochon (1993) pour choisir les cinq enseignants experts qui ont participé à la recherche. En effet, malgré la richesse des recherches sur la question, la définition de l'enseignant expert est encore problématique (Visioli \& Ria, 2010), et le recours, tel que le propose l'auteur, à des critères multiples (expérience professionnelle, connaissance didactique, connaissance pédagogique, pratique réflexive, reconnaissance sociale) paraît être une solution garantissant une certaine rigueur méthodologique, dans l'attente d'une éventuelle théorie scientifique de l'expertise enseignante (Tableau I). 


\section{eJRIEPS 40 janvier 2017}

Tableau I. Caractéristiques principales des enseignants participant à la recherche.

\begin{tabular}{llllll}
\hline Prénom & Age & $\begin{array}{l}\text { Ancienneté } \\
\text { comme } \\
\text { enseignant }\end{array}$ & $\begin{array}{l}\text { Formateurs } \\
\text { stages } \\
\text { académiques }\end{array}$ & $\begin{array}{l}\text { Niveau du } \\
\text { concours de } \\
\text { recrutement }\end{array}$ & $\begin{array}{l}\text { Spécialité } \\
\text { sportive }\end{array}$ \\
\hline Christophe & 52 ans & 29 ans & Oui & $\begin{array}{l}\text { Agrégation } \\
\text { EPS }\end{array}$ & $\begin{array}{l}\text { Activités de } \\
\text { raquette }\end{array}$ \\
\hline Catherine & 48 ans & 27 ans & $\begin{array}{l}\text { Oui } \\
\text { Formatrice } \\
\text { PLC2 }\end{array}$ & $\begin{array}{l}\text { Agrégation } \\
\text { EPS }\end{array}$ & $\begin{array}{l}\text { Sports } \\
\text { collectifs }\end{array}$ \\
\hline François & 55 ans & 30 ans & Non & CAPEPS & $\begin{array}{l}\text { Activités de } \\
\text { raquette }\end{array}$ \\
\hline Philippe & 60 ans & 39 ans & Oui & $\begin{array}{l}\text { Agrégation } \\
\text { EPS }\end{array}$ & $\begin{array}{l}\text { Sports } \\
\text { collectifs \& } \\
\text { Sports de } \\
\text { raquette }\end{array}$ \\
\hline Paul & 36 ans & 10 ans & Oui & CAPEPS & $\begin{array}{l}\text { Sports } \\
\text { collectifs }\end{array}$ \\
\hline
\end{tabular}

Chaque enseignant a été observé au cours de deux leçons, avec des classes de collège (sixième, cinquième) et de lycée (seconde, première), et des Activités Physiques Sportives et Artistiques (APSA) variées (badminton, tennis de table, danse, arts du cirque). Seul Christophe a été observé durant une seule leçon (Tableau II).

Tableau II. Caractéristiques principales des situations étudiées

\begin{tabular}{lllll}
\hline Prénom & $\begin{array}{l}\text { Classes } \\
\text { observées }\end{array}$ & $\begin{array}{l}\text { APSA } \\
\text { observées }\end{array}$ & Spécialiste & $\begin{array}{l}\text { Temps de cours } \\
\text { effectif }\end{array}$ \\
\hline Christophe & $1^{\text {ère }}$ & Tennis de table & Oui & $1 \mathrm{~h} 52$ \\
\hline \multirow{2}{*}{ Catherine } & $2^{\text {nd }}$ & Danse & Non & $1 \mathrm{~h} 50$ \\
\cline { 2 - 5 } & $1^{\text {ère }}$ & Badminton & Oui & $1 \mathrm{~h} 48$ \\
\hline \multirow{2}{*}{ François } & $2^{\text {nd }}$ & Badminton & Oui & $1 \mathrm{~h} 53$ \\
\cline { 2 - 5 } & $1^{\text {ère }}$ & Arts du cirque & Non & $1 \mathrm{~h} 46$ \\
\hline \multirow{2}{*}{ Philippe } & $6^{\text {ème }}$ SEGPA & Danse & Non & $1 \mathrm{~h} 35$ \\
\hline \multirow{2}{*}{ Paul } & $6^{\text {ème }}$ SEGPA & Badminton & Oui & $1 \mathrm{~h} 30$ \\
\cline { 2 - 5 } & $6^{\text {ème }}$ & Danse & Non & $1 \mathrm{~h} 18$ \\
\hline
\end{tabular}




\section{eJRIEPS 40 janvier 2017}

Les conditions de la collaboration entre participants et chercheur ont été précisées dès le départ de la recherche (information des objectifs de l'étude et des conditions de participation, garantie de confidentialité et exploitation des résultats avec l'aval des acteurs, compte rendu des avancées de la recherche). A noter que l'instauration d'une relation de confiance mutuelle entre le chercheur et les participants a été une préoccupation centrale tout au long de l'étude, pour rendre possible l'explicitation de l'activité, en particulier celle des émotions.

\section{2. Recueil des données}

Pour chacune des leçons, trois catégories de données ont été systématiquement recueillies :

(1) des données d'enregistrement des enseignants et des élèves en situation réelle, obtenues à l'aide d'une caméra numérique sur pied et d'un micro émetteur HF porté par l'enseignant, et recueillies en continu, de l'arrivée dans le gymnase au départ des élèves aux vestiaires, afin de restituer le caractère dynamique du cours d'action ;

(2) des données a posteriori, c'est-à-dire l'enregistrement vidéo des verbalisations lors d'un EAC, au cours duquel chaque enseignant a été invité à expliciter, montrer et commenter les éléments significatifs pour lui de la leçon, en présence d'un interlocuteur. Ces entretiens ont repris la chronologie des situations considérées, mais les relances effectuées par le chercheur ne concernaient pas toutes les actions réalisées, et portaient principalement sur les actions qui avaient été émotionnellement significatives (et par conséquent décrites et commentées) pour les enseignants ;

(3) l'auto-estimation des émotions sur une échelle de «Plaisir - Déplaisir » composée de onze points (de -5 : extrêmement déplaisant à +5 : extrêmement plaisant) similaire à celle utilisée par Visioli et al. (2014). Pendant l'EAC, les enseignants devaient estimer leur plaisir - déplaisir régulièrement tout au long de la leçon. Plus précisément, cette autoestimation s'est produite quand : (a) l'enseignant intervenait spontanément pour commenter une de ses actions ; (b) le chercheur intervenait et/ou relançait l'enseignant ; (c) en cas de non intervention de l'enseignant ou du chercheur durant un laps de temps supérieur à une minute.

Cette échelle a constitué un instrument de mesure de la dynamique émotionnelle tout au long des leçons d'EPS, afin de les associer aux contenus significatifs pour les acteurs, et plus largement de comprendre leur rôle au sein de l'activité humaine. Il est à noter que les données quantitatives recueillies ont un statut secondaire dans notre recherche par rapport aux données qualitatives recueillies durant les EAC. Leur intérêt figure dans la 


\section{eJRIEPS 40 janvier 2017}

possibilité qu'elles offrent de rendre compte sous forme graphique, et donc plus visuelle, la dynamique émotionnelle des enseignants, et dans le point d'appui qu'elles procurent aux enseignants dans le processus d'explicitation de leurs émotions.

\section{3. Traitement des données}

Les données ont été traitées en six étapes:

(1) la construction de protocoles à deux volets (Tableau III) présentant d'une part la description des comportements et des verbalisations des enseignants et des élèves, et d'autre part les données d'EAC ainsi que les valeurs sur l'échelle de Plaisir - Déplaisir.

Tableau III. Extrait d'un protocole à deux volets (leçon de danse de Catherine)

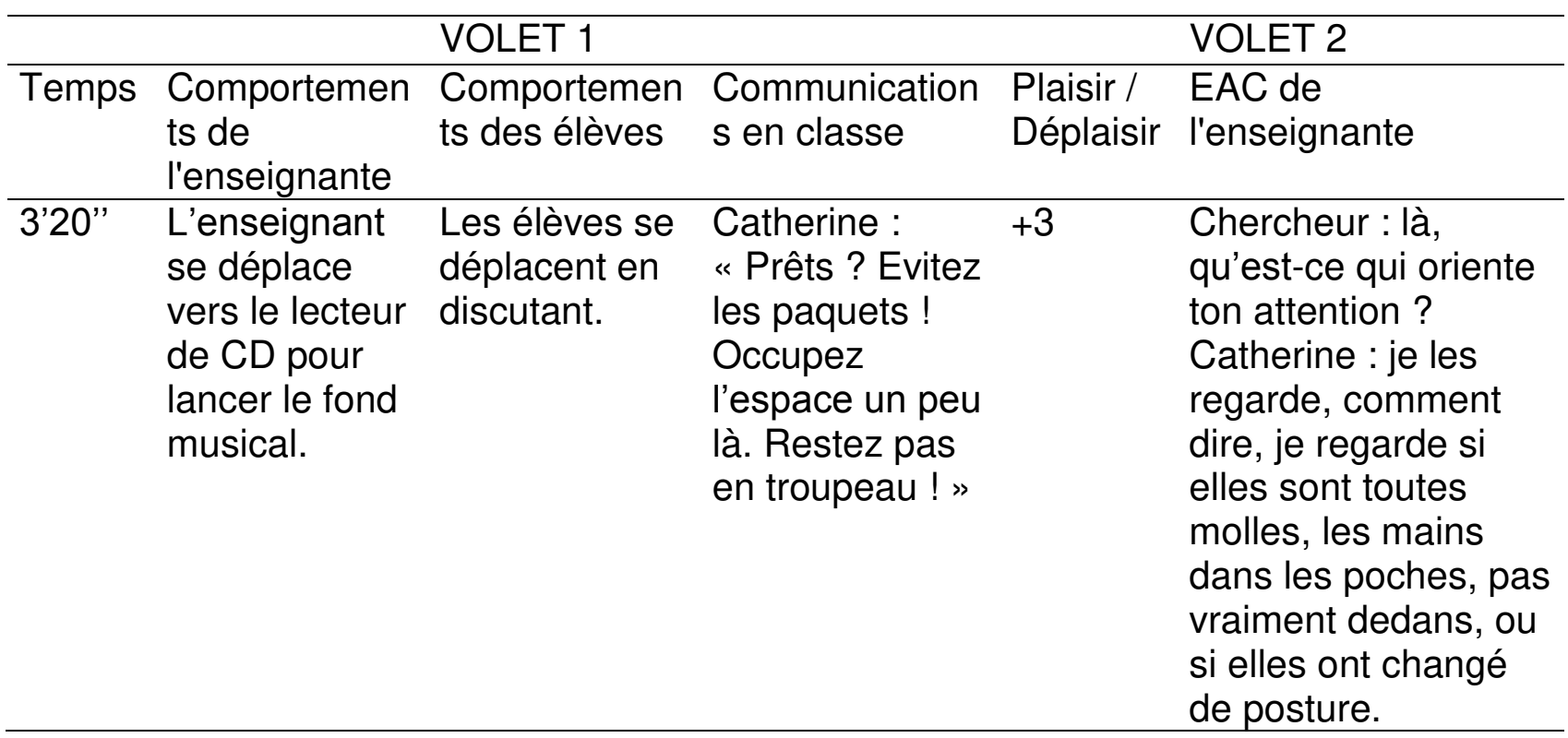

(2) la documentation du signe hexadique (Theureau, 2006), qui décrit la conscience préréflexive d'un acteur donné à un instant $t$, sur la base de six composantes, chacune décrivant une catégorie de l'expérience de l'acteur à chaque instant de son activité : (a) l'unité significative élémentaire $(U)$ du cours d'expérience est la fraction de l'activité qui est montrée, racontée et commentée par l'acteur. Elle peut être une interprétation ou une action en lien avec les émotions ressenties par l'enseignant et sa disponibilité à un instant $t$; (b) l'engagement (E) dans la situation a trait aux préoccupations de l'enseignant dans la situation, notamment associées à ses émotions ressenties dans l'interaction avec les élèves. II témoigne aussi d'une disponibilité particulière auprès d'eux ; (c) le representamen $(R)$ est ce qui fait signe pour l'acteur dans la situation. II peut être un jugement perceptif, mnémonique ou proprioceptif, exprimant par exemple les causes et les effets des émotions de l'enseignant. II renseigne aussi, notamment, sa disponibilité à 


\section{eJRIEPS 40 janvier 2017}

percevoir l'environnement ; (d) l'actualité potentielle (A) regroupe ce qui est attendu par l'acteur dans la situation, compte tenu de l'engagement, et peut venir illustrer ce que s'attend à obtenir l'enseignant comme résultat lorsqu'il ressent des émotions particulièresface aux élèves. Elle peut être plus ou moins orientée vers l'activité des élèves et témoigner ainsi de la disponibilité de l'enseignant auprès d'eux ; (e) le référentiel (S) a trait aux éléments de connaissance issus des cours d'expérience passés et mobilisés par l'acteur à un instant $t$. Ces connaissances appartiennent à sa culture, et peuvent être liées eux émotions de l'acteur en classe et à sa disponibilité auprès des élèves ; (f) l'interprétant (I) est la construction d'éléments de connaissance (validation ou invalidation) de l'acteur dans la situation, et éclaire par exemple les apprentissages concomitants à ses émotions ressenties et à sa disponibilité en classe.

(3) la modélisation de la dynamique émotionnelle des enseignants (succession de données auto-estimées sur l'échelle plaisir-déplaisir), à l'aide d'un graphique croisant les valeurs auto-estimées par les enseignants et le temps du cours.

(4) le calcul de moyennes, d'écarts-type, de valeurs positives et négatives maximales des valeurs auto-estimées par chaque enseignant lors des cours. Ce calcul permet de dresser des conclusions générales quant à la stabilité, l'intensité et la valence des émotions ressenties par les enseignants. II offre également la possibilité d'opérer des comparaisons inter-cas.

(5) la modélisation inter-cas de l'activité des enseignants d'EPS experts. L'identification de ces régularités a été effectuée par un processus de comparaison des études de cas, dans un premier temps pour un même enseignant, puis dans un second temps en reprenant l'ensemble des modélisations intermédiaires afin de réaliser une modélisation globale de l'activité des enseignants experts d'EPS. Cette comparaison a abouti à une classification a posteriori des éléments typiques de l'activité des enseignants en classe en lien avec leur dynamique émotionnelle (Tableau IV). 


\section{eJRIEPS 40 janvier 2017}

Tableau IV. Classification a posteriori des connaissances typiques mobilisées par les enseignants en lien avec leur dynamique émotionnelle en classe

\begin{tabular}{|c|c|c|}
\hline Catégorie & $\begin{array}{l}\text { Sous- } \\
\text { catégorie }\end{array}$ & Extrait d'EAC typique \\
\hline \multirow[t]{2}{*}{$\begin{array}{l}\text { Les } \\
\text { connaissances } \\
\text { sur soi }\end{array}$} & $\begin{array}{l}\text { Les } \\
\text { connaissances } \\
\text { sur ses } \\
\text { propres } \\
\text { valeurs }\end{array}$ & $\begin{array}{l}\text { «ben c'est à dire que je veux arriver à cet état où je } \\
\text { peux prendre la parole dans un climat où je pense } \\
\text { que les élèves peuvent m'écouter. C'est à dire pour } \\
\text { moi c'est le silence (...) je trouve que c'est un manque } \\
\text { de respect, et je leur dis souvent, moi quand tu me } \\
\text { parles, je t'écoute, je ne suis pas en train de faire } \\
\text { autre chose... Cette égalité relationnelle, j'insiste } \\
\text { toujours là-dessus » (Paul, EPD: -1). }\end{array}$ \\
\hline & $\begin{array}{l}\text { Les } \\
\text { connaissances } \\
\text { sur } \\
\text { l'actualisation } \\
\text { de ces valeurs } \\
\text { en situation }\end{array}$ & $\begin{array}{l}\text { " Je le prends relativement tranquillement parce que } \\
\text { ce sont des choses qui arrivent, surtout en début de } \\
\text { séance ou tout se met en route, ça arrive très } \\
\text { fréquemment. En début de séance ou lors du } \\
\text { lancement d'une tâche. Donc l'expérience m'a appris } \\
\text { à gérer tout ça sans paniquer particulièrement " } \\
\text { (Christophe, EPD :-1). }\end{array}$ \\
\hline
\end{tabular}

L'identification du caractère typique de l'activité analysée repose sur trois critères : (a) l'énonciation par les enseignants dans l'EAC du caractère typique de leur activité associée à une situation récurrente; (b) la documentation d'une configuration typique des composantes des signes hexadiques ; (c) la fréquence d'occurrence de ces configurations typiques des composantes des signes hexadiques au niveau d'un même cours d'action, ou au niveau des cours d'action de plusieurs enseignants. Par la suite, la comparaison des cas intra individuels et inter individuels a permis de monter d'un niveau supplémentaire en termes de généralisation des résultats, pour aboutir à l'identification de catégories inter cas concernant la dynamique émotionnelle des enseignants et les relations avec la disponibilité auprès des élèves.

(6) plusieurs procédures ont été utilisées pour garantir la validité du traitement des données. Premièrement, les transcriptions des données ont été présentées aux enseignants pour s'assurer de l'authenticité des commentaires et pour permettre d'éventuelles modifications. Deuxièmement, les signes hexadiques ont été documentés par un enseignant doctorant et un enseignant chercheur possédant tous deux une forte expérience professionnelle de l'enseignement de l'EPS. Leur agrément initial a été de $85 \%$. Chaque point de désaccord a fait l'objet d'une discussion de manière à parvenir à un taux d'agrément de $100 \%$. 


\section{eJRIEPS 40 janvier 2017}

\section{Résultats}

L'objectif de cette étude était d'analyser la dynamique émotionnelle d'enseignants experts lors de cours d'EPS. Les résultats obtenus au cours de cette recherche sont structurés en trois parties, rapportant chacune des exemples typiques de l'activité des enseignants lors des leçons: (1) trois constats concernant la dynamique émotionnelle des enseignants d'EPS experts ; (2) la dynamique émotionnelle et les connaissances mobilisées lors du cours ; (3) la dynamique émotionnelle et la disponibilité auprès des élèves.

4. 1. Trois constats concernant la dynamique émotionnelle des enseignants d'EPS experts

La figure 1 réunit l'ensemble des dynamiques émotionnelles des enseignants lors des cours d'EPS. Pour illustration, nous avons modélisé sur un graphique des extraits de signes hexadiques (engagement). 

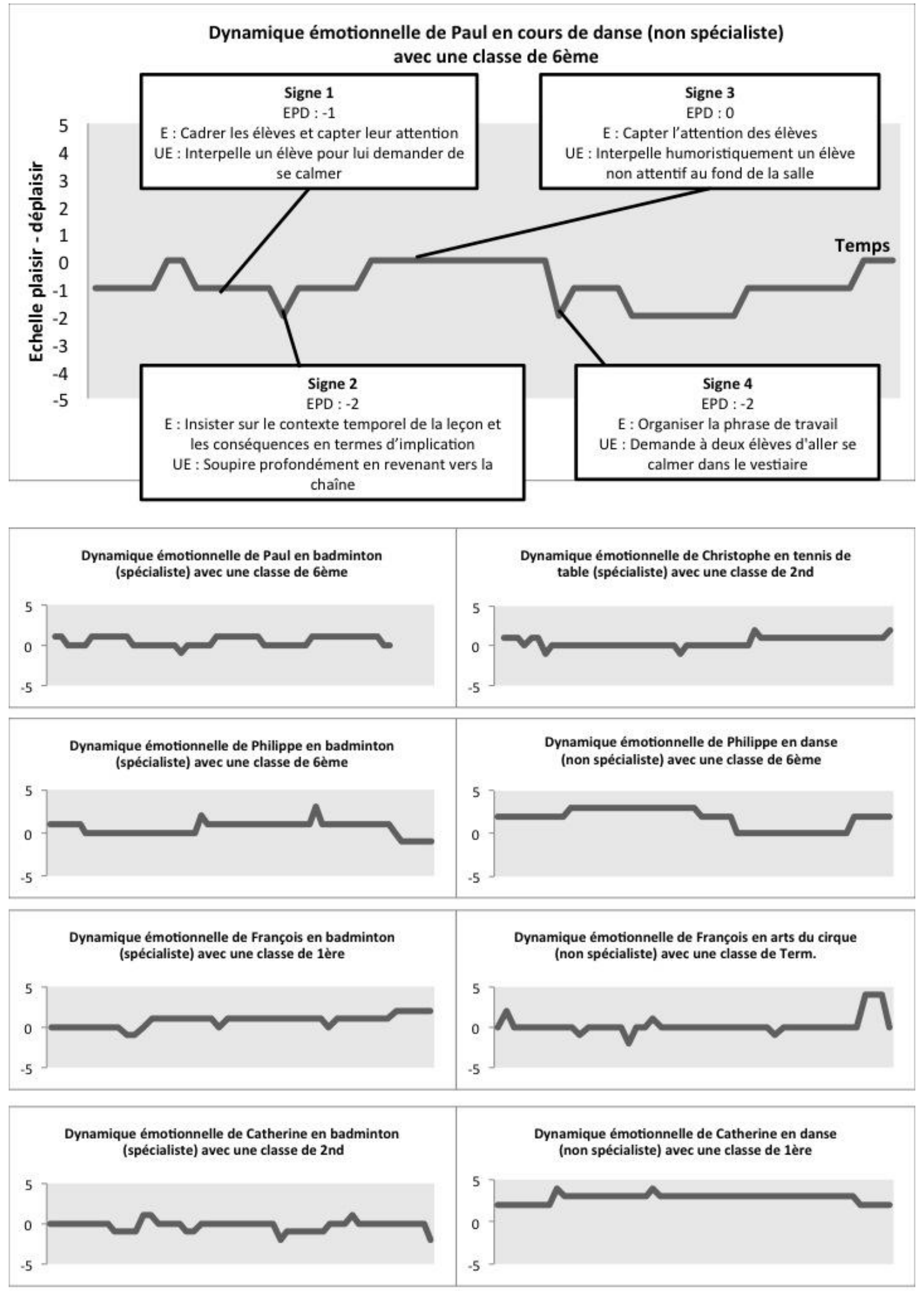

Figure 1. Dynamique émotionnelle des enseignants experts lors des situations analysées 


\section{eJRIEPS 40 janvier 2017}

Le tableau $\mathrm{V}$ rapporte les données chiffrées permettant de réaliser des conclusions générales sur la stabilité, l'intensité et la valence des émotions ressenties par les enseignants.

Tableau V. Moyennes, écarts-types, valeurs minimales et valeurs maximales des dynamiques émotionnelles des enseignants experts

\begin{tabular}{|c|c|c|c|c|c|c|c|}
\hline & APSA & $\begin{array}{l}\text { Spécialist } \\
\mathrm{e}\end{array}$ & Classe & $\begin{array}{l}\text { Valeur } \\
\text { minimum }\end{array}$ & $\begin{array}{l}\text { Valeur } \\
\text { maximum }\end{array}$ & $\begin{array}{l}\text { Ecart- } \\
\text { type }\end{array}$ & Moyenne \\
\hline \multirow[b]{2}{*}{ Paul } & Danse & Non & Danse & -2 & 0 & 0,69 & $-0,87$ \\
\hline & $\begin{array}{l}\text { Tennis de } \\
\text { table }\end{array}$ & Oui & $\begin{array}{l}\text { Tennis de } \\
\text { table }\end{array}$ & -1 & 1 & 0,54 & 0,51 \\
\hline \multirow[b]{2}{*}{ Catherine } & Danse & Non & Danse & 2 & 4 & 0,49 & 2,79 \\
\hline & $\begin{array}{l}\text { Badminto } \\
\mathrm{n}\end{array}$ & Oui & $\begin{array}{l}\text { Badminto } \\
\mathrm{n}\end{array}$ & -2 & 1 & 0,61 & $-0,24$ \\
\hline \multirow{2}{*}{ François } & $\begin{array}{l}\text { Arts du } \\
\text { Cirque }\end{array}$ & Non & $\begin{array}{l}\text { Arts du } \\
\text { cirque }\end{array}$ & -2 & 4 & 1,08 & 0,22 \\
\hline & $\begin{array}{l}\text { Badminto } \\
\mathrm{n}\end{array}$ & Oui & $\begin{array}{l}\text { Badminto } \\
\mathrm{n}\end{array}$ & -1 & 2 & 0,7 & 0,76 \\
\hline \multirow[b]{2}{*}{ Philippe } & Danse & Non & Danse & 0 & 3 & 1,2 & 1,74 \\
\hline & $\begin{array}{l}\text { Badminto } \\
\mathrm{n}\end{array}$ & Oui & $\begin{array}{l}\text { Badminto } \\
\mathrm{n}\end{array}$ & -1 & 3 & 0,6 & 0,71 \\
\hline $\begin{array}{l}\text { Christoph } \\
\text { e }\end{array}$ & $\begin{array}{l}\text { Tennis de } \\
\text { table }\end{array}$ & Non & $\begin{array}{l}\text { Tennis de } \\
\text { table }\end{array}$ & -1 & 2 & 0,6 & 0,4 \\
\hline
\end{tabular}

Ces éléments quantitatifs permettent d'aboutir à trois constats concernant la dynamique émotionnelle des enseignants experts : (1) une dynamique émotionnelle majoritairement positive; (2) une dynamique émotionnelle parfois négative; (3) une dynamique émotionnelle stable et modérée.

4. 1. 1. Des hausses de la dynamique vers des émotions positives En premier lieu, la dynamique émotionnelle des enseignants présente des moments de forte hausse lors de certaines leçons. Par exemple, lors du cours de Cirque, François ressent des émotions positives intenses à partir du moment où son engagement est d'aider deux élèves à trouver une solution pour réaliser une figure qu'il ne connaît pas non plus : «Ben là, c'est +4 , mais sans en avoir réellement conscience. En fait, je suis tellement dans la réflexion, et puis les deux gamins essayent, on est ensemble tous les trois à chercher (...) il n'y a plus vraiment de prof, c'est juste trois personnes qui essayent de résoudre un problème » (EAC François, arts du cirque). L'enseignant accepte de s'immerger, avec les élèves, dans une situation incertaine. Son activité s'apparente ici à de l'improvisation et génère chez lui un plaisir intense. Dans un autre exemple, l'analyse 


\section{eJRIEPS 40 janvier 2017}

du cours d'action de Catherine en danse montre également que sa dynamique émotionnelle connaît de fortes hausses. Au sujet de la fin de l'échauffement, un regroupement a lieu et une élève chute. L'enseignant déclare : « tu vois, lorsqu'elle se casse la figure, on est tous en train de se marrer. C'est vraiment agréable de travailler dans cette ambiance » (EAC Catherine, danse). A partir de ce moment, elle ressent un plaisir très intense, à hauteur de +4 sur l'EPD. Nous constatons que la majorité des constats de fortes hausses dans la dynamique émotionnelle des enseignants ont lieu lors de leçon de danse ou d'arts du cirque, où les enseignants manifestent un niveau faible de spécialisation. Un écart-type élevé est alors à noter, révélant un décalage relativement important entre les valeurs minimales et maximales recueillies dans ces APSA (Tableau V). Nous pouvons faire l'hypothèse que ces APSA sont génératrices d'émotions positives intenses liées notamment à la nouveauté, malgré des diminutions en termes de dynamique émotionnelle.

\section{1. 2. Des baisses de la dynamique vers des émotions négatives}

En second lieu, l'analyse de la dynamique émotionnelle des enseignants experts témoigne également de baisses, même si celles-ci sont moins importantes que les hausses. La figure 1 illustre des pics sur la courbe de leurs dynamiques émotionnelles, traduisant des émotions plus intenses. Par exemple, Catherine estime en cours de badminton des émotions négatives jusqu'à -2 en raison de l'absence de réaction de ses élèves lors d'un regroupement en cours de badminton : «Pas de réaction! c'est terrible ça ! Je fais mon travail, je raconte mon truc, mais je ne sais pas quel effet ça a, j'ai pas de retour (...) II y a un silence pesant! Ils attendent que ça se passe» (EAC Catherine, badminton). L'engagement de l'enseignante est de générer une certaine émulation au sein de la classe, mais selon elle, elle n'y parvient pas. Dans un autre exemple, François ressent des émotions négatives car il connaît des difficultés à répondre aux sollicitations d'élèves en arts du cirque : "là, elles me posent la question de savoir ce que l'on peut faire avec ça. Et là, je commence à pas être à l'aise. J'ai essayé au cycle précédent, je suis pas bon làdedans, donc là je vais chercher mes lunettes, c'est peut être aussi pour gagner du temps (...) donc là, c'est -2. Par contre, je sais que ça va bien se passer avec les élèves » (EAC François, arts du cirque). Toutefois, ces émotions négatives ne sont pas décrites par les enseignants comme très intenses, et ne sont jamais estimées plus bas que -2 sur l'EPD (Tableau V). Dans l'exemple de François, le manque de maitrise de l'APSA est compensé par son référentiel concernant la qualité de sa relation avec les élèves.

4. 1. 3. Une dynamique émotionnelle stable et modérée 


\section{eJRIEPS 40 janvier 2017}

L'analyse des émotions ressenties par les enseignants lors des cours a permis de mettre en évidence une dynamique émotionnelle stable et modérée des enseignants experts. En effet, la moyenne se situe dans 7 cas sur 9 entre -1 et 1 , ce qui témoigne d'une certaine modération des émotions ressenties en moyenne par les enseignants. Aussi, l'écart-type se situe 7 fois sur 9 entre 0 et 1 , ce qui témoigne d'une stabilité des émotions des experts autour de valeurs neutres. L'analyse du cours d'action des enseignants montre qu'ils font preuve d'un certain recul contribuant au caractère plutôt positif, modéré et stable de leur dynamique émotionnelle, en dépit des évènements non souhaités se produisant en contexte. Par exemple, au milieu du cours de badminton, Catherine réalise un effort de prise de recul lui permettant de ne pas trop s'impliquer affectivement devant le manque d'engagement qu'elle dit percevoir chez ses élèves : “Au feeling, je suis à zéro (...) c'est un truc neutre, il ne se passe rien, avec des petits moments où elles me gonflent un peu, mais je pense que l'expérience me permet de mettre suffisamment de distance pour ne pas me laisser embarquer (...) II se passe des choses, mais quelque part, c'est un peu extérieur à moi. Je pense que je me positionne à un moment avec ces élèves dans un truc ou je me protège, parce que sinon je vais me foutre une balle parce que je n'arrive pas à les faire bouger »(EAC Catherine, badminton). Ses états affectifs sont auto-estimés à 0 alors que l'enseignante a pour préoccupation de favoriser un engagement plus soutenu des élèves dans l'activité.

Un des indicateurs de cette prise de recul est l'humour dont font fréquemment preuve les enseignants en classe, alors que leur préoccupation réside dans le recadrage des élèves. Par exemple, au début du cours de danse, Paul est contraint de changer de salle au dernier moment et se trouve confronté à des élèves très chahuteurs. Malgré cela, il déclare durant l'EAC : « c'est stable, mais je me dis que ça va être long (...). Je sens que c'est difficile, mais je m'accroche. Je m'accroche, j'y mets quand même du mien » (EAC Paul, danse, EPD : -1). Lors de cette situation, alors que son représentamen est un élève qui se regarde dans la glace au lieu d'écouter les consignes, il utilise l'humour pour le recadrer. A cet instant, il estime que son plaisir-déplaisir passe de -1 à 0 (Signe 3) : « $A h$ John! Je sais que tu es face à la glace, que tu aimes bien te regarder dedans... c'est pour ça que je t’ai mis là ».

4. 2. Une dynamique émotionnelle stable, modérée et positive en lien avec les connaissances mobilisées par l'enseignant

Les enseignants experts en EPS établissent spontanément le lien entre leurs états affectifs ressentis en classe et certaines connaissances qu'ils mobilisent dans la situation. 


\section{eJRIEPS 40 janvier 2017}

La classification de ces connaissances a posteriori a permis de mettre en évidence trois catégories de connaissances qui participent de leur dynamique émotionnelle stable, modérée et plutôt positive : (1) des connaissances sur soi ; (2) des connaissances sur les élèves ; (3) des connaissances sur l'APSA.

\section{2. 1. Les connaissances sur soi}

Tout d'abord, les enseignants experts mobilisent en situation des connaissances sur soi, qui participent de leur dynamique émotionnelle stable et modérée. Ce référentiel est de deux ordres. Premièrement, les enseignants experts mobilisent des connaissances sur leurs propres valeurs. Ces connaissances leur confèrent une véritable conception de I'EPS et une certaine clarté dans leurs attentes vis-à-vis des élèves. Par exemple, Catherine exprime la conviction inébranlable que les élèves présentent une chorégraphie de danse à la fin du cours : “c'est non négociable. La manière dont je lui réponds, c'est clair. C'est non négociable, et quoi qu'il arrive, ce sera aujourd'hui (...) moi je sais, dans mon discours je sais ce que je veux 》 (EAC Catherine, danse, EPD : +2).

Deuxièmement, les enseignants experts mobilisent également des connaissances liées à l'actualisation de ces valeurs en situation de classe. Autrement dit, s'ils expriment des convictions sur leurs propres valeurs, ils savent également que le contexte de classe implique une nécessaire souplesse, ce qui les amène à ne pas se montrer trop pris au dépourvus devant les évènements en classe. Par exemple, Christophe sait que ses valeurs liées au cadrage des élèves doivent être assouplies en début de séance : « Je le prends relativement tranquillement parce que ce sont des choses qui arrivent, surtout en début de séance ou tout se met en route, ça arrive très fréquemment. (...) L'expérience m'a appris à gérer tout ça sans paniquer particulièrement » (EAC Christophe, tennis de table, EPD : -1).

\section{2. 2. Les connaissances sur les élèves}

Par ailleurs, les enseignants experts mobilisent en situation des connaissances sur les élèves, qui participent de leur dynamique émotionnelle stable, modérée et positive. Ce référentiel est de deux ordres. Premièrement, ces connaissances ont trait au rapport des élèves à la pratique des APSA. Philippe mobilise par exemple des connaissances sur les rapports de Melissa aux APSA et notamment sur ses problèmes de coordination : “ c'est une élève qui ne voit pas beaucoup et qui en plus à des problèmes de coordination. Dans les jeux y compris avec des gros ballons, volley, handball, elle a beaucoup de mal à attraper, ce qui concerne l'anticipation coïncidence » (EAC Philippe, badminton, EPD : 


\section{eJRIEPS 40 janvier 2017}

+1). Ces connaissances amènent l'enseignant à ne pas être surpris et désemparé face aux difficultés connues par l'élève durant le cours.

Deuxièmement, les connaissances des enseignants en lien avec les élèves ont également trait à leur personnalité. C'est par exemple le cas de Christophe au sujet de Yunus : «il (Yunus) peut avoir des réactions très épidermiques, c'est ce qui s'est passé au premier trimestre (...) Yunus c'est un gamin que je connais bien " (EAC Christophe, tennis de table, EPD : +2). Connaître la personnalité de l'élève permet à l'enseignant d'anticiper certaines conduites déviantes, et participe ainsi de leur dynamique émotionnelle modérée, stable et plutôt positive.

\section{2. 3. Les connaissances sur l'APSA}

Par ailleurs, les enseignants experts mobilisent en situation des connaissances sur l'APSA, qui participent de leur dynamique émotionnelle stable, modérée et positive. Ce référentiel est de deux ordres. En premier lieu, les enseignants experts mobilisent des connaissances spécifiques à l'APSA. Par exemple, au début du cours de tennis de table, Christophe mobilise des connaissances sur l'APSA alors qu'il mime certains coups techniques. Ces connaissances l'amènent à se sentir plus crédible et à ressentir des émotions plus positives en cours: «moi j'estime qu'un prof d'EPS doit s'impliquer physiquement à certains moments, pour avoir une certaine crédibilité. Alors je le fais pas systématiquement dans toutes les APS mais comme en tennis de table j'en ai fait en club, que lors de séances précédentes, je leur ai montré certains aspects techniques, je peux me permettre d'illustrer sans adversaire en face » (EAC Christophe, tennis de table, EPD : $+1)$.

En second lieu, les enseignants experts mobilisent des connaissances sur les APSA plus transversales, à l'image de François qui, en arts du cirque, mobilisent des connaissances liées au ski et au surf, mais aussi des connaissances plus méthodologiques sur les manières de réfléchir dans les APSA. Ces connaissances alimentent le jeu de l'enseignant avec les élèves et génèrent des émotions positives, en dépit du manque de spécialisation de François dans l'APSA : « là, je leur dis de passer d'un appui sur l'autre. Donc là, je me réfère à ce que je connais, le ski, le surf... Donc là je vais chercher des connaissances que j'ai : par exemple, si on ne décale pas un appui, ça ne marche pas (...) je suis plutôt content parce que c'est un jeu pour moi » (EAC François, arts du cirque, EPD : +4).

4. 3. Une dynamique émotionnelle stable, modérée et positive qui participe de la disponibilité de l'enseignant auprès des élèves 


\section{eJRIEPS 40 janvier 2017}

Si les résultats ne permettent pas d'établir de rapports d'influence entre dynamique émotionnelle et disponibilité de l'enseignant, reste qu'ils font apparaître une association entre une dynamique émotionnelle plutôt modérée, stable et positive des enseignants experts en EPS et une certaine disponibilité aux élèves lors des cours. Cette disponibilité se traduit de deux manières : (1) percevoir finement l'activité des élèves ; (2) exprimer des préoccupations dirigées vers les élèves.

\section{3. 1. Percevoir finement l'activité des élèves}

La disponibilité des enseignants experts s'illustre dans un premier temps dans leur capacité à percevoir l'activité des élèves durant le cours. Cette activité perceptive est associée à une dynamique émotionnelle modérée, stable et plutôt positive des experts. Tout d'abord, les enseignants experts perçoivent finement le niveau d'engagement des élèves dans la pratique des APSA. Par exemple, Catherine explicite ses perceptions au début du cours, alors qu'elle estime son plaisir à +2 : « Je les regarde, comment dire, je regarde si elles sont toutes molles, les mains dans les poches, pas vraiment dedans, ou si elles ont changé de posture. Ce qui est pour moi un indice sur « on rentre dedans ». On se déplace, on marche, on écoute, on essaye d'être dans le rythme, etc... au départ, c'est ça que je regarde, sur le début de séance » (EAC Catherine, danse, EPD : +2).

Par ailleurs, les enseignants experts parviennent à percevoir finement le niveau d'attention des élèves en cours. Par exemple, l'analyse du cours d'action de Christophe en tennis de table montre qu'il est très attentif aux comportements des élèves témoignant de leur niveau d'attention en cours: « pour l'instant, quand je regarde les visages, les mouvements ou plutôt le manque de mouvements, il me semble qu'ils sont assez centrés sur le tableau, je ressens ça comme plutôt positif. II me semble que le climat est plutôt à l'écoute, alors que le passage de l'appel n'est pas toujours un passage facile » (EAC Christophe, tennis de table, EPD: +1). La disponibilité de Christophe est également associée à une dynamique émotionnelle plutôt modérée, stable et positive qui n'accapare pas son attention.

Enfin, les enseignants experts se montrent disponibles à l'état émotionnel des élèves. Par exemple, lors du regroupement initial en cours de badminton, lorsqu'un élève arrive en retard, François se montre particulièrement disponible à lui : « là, je vois son attitude, il est tête basse, bon il sait qu'il a fait une connerie (...) II marque le coup quelque part : la tête en bas, il bafouille, il me rentre pas dedans par exemple. Là il parle plus, le gamin il est un petit peu perdu, donc là ça me dérange» (EAC François, badminton, EPD : -1). Son representamen est lié à son attitude, ses préoccupations et ses émotions. 


\section{eJRIEPS 40 janvier 2017}

4. 3. 2. Exprimer des préoccupations dirigées vers les élèves

Dans un second temps, la disponibilité de François s'illustre à travers ses préoccupations en cours d'action, particulièrement tournées vers les élèves. Premièrement, les experts ont pour préoccupation de soutenir les élèves. Par exemple, François est préoccupé par le soutien de l'élève en retard, chez qui il a perçu un certain mal-être : « là tout d'un coup je commence quand même à me poser des questions, parce que j'ai mis le cadre législatif, et là je vais rentrer dans le cadre personnel une attitude comme celle-là peut être révélatrice de quelque chose. Et là, j'essaye de voir si il n'y a pas quelque chose, tout en lui disant qu'il n'est pas forcément obligé de m'en parler » (EAC François, arts du cirque, EPD : +1). Nous remarquons que ces préoccupations sont associées à des émotions stables, modérées et positives.

Deuxièmement, les experts ont la préoccupation de négocier avec les élèves, à l'image de Philippe avec Giovanni, qui exprime avec provocation s'ennuyer dans la situation en badminton. L'enseignant lui répond: « ouais mais tout à l'heure, tu vas pas... si tu t'ennuies, c'est que tu veux pas faire le bon entraîneur. C'est vrai que ce n'est pas facile pour toi mais tout à l'heure tu vas jouer, je te promets, tu vas voir, dans 5-6 minutes ». Durant I'EAC, Philippe exprime la préoccupation de négocier avec l'élève, de ne pas le blesser et en vient même à remettre en cause cette situation dont il fait fréquemment appel : « ben là il m'a questionné. (...) Mais il a raison quelque part. II a raison il m'a dit je m'ennuie. Quelque part ça va peut-être me faire revenir sur cette procédure que j'utilise assez souvent, depuis longtemps d'ailleurs » (EAC Philippe, badminton, EPD : -2). Cette situation, bien qu'inconfortable, ne génère pas d'émotions négatives très intenses chez l'enseignant.

Enfin, les enseignants experts ont également la préoccupation d'imposer aux élèves certaines règles, dans leur intérêt. Ces préoccupations sont associées à une dynamique émotionnelle plutôt stable, modérée et positive en cours d'EPS. Par exemple, Catherine a la préoccupation d'imposer aux élèves de présenter leur chorégraphie à la fin du cours de danse, pour les aider à finaliser le travail et les dynamiser : « et puis en même temps, ça les excite, ça fait peur mais en même temps ça finalise vraiment le travail sinon... donc il y a cette ambiguïté : on a la trouille mais en même temps... et c'est aussi quelque chose qui les met en action » (EAC Catherine, danse, EPD : +2).

\section{Discussion}




\section{eJRIEPS 40 janvier 2017}

La discussion est structurée en quatre parties, chacune formulée sous la forme d'une question que nos résultats permettent d'éclairer : (1) la dynamique émotionnelle des enseignants : un plaisir d'enseigner ? ; (2) la dynamique émotionnelle des enseignants d'EPS : quelles différences entre les experts et les novices ? ; (3) entre sentiment de contrôle et prise de distance des enseignants: quels processus de régulation émotionnelle en situation ? ; (4) quelles conséquences sur la formation initiale et continue des enseignants?

5. 1. La dynamique émotionnelle des enseignants : un plaisir d'enseigner ?

Les résultats obtenus au cours de cette étude font état d'une dynamique émotionnelle relativement stable et modérée, mais également positive des enseignants experts, nous permettant de discuter du plaisir d'enseigner l'EPS et de porter un regard relativement optimiste sur cette profession. Certains d'entre eux comme François et Catherine ont ressenti un plaisir élevé lors de leur leçon d'arts du cirque et de danse, notamment en lien avec la qualité des interactions avec les élèves. A ce titre, un rapport intitulé «Enseigner, un métier pour demain » (Obin, 2002), souligne que « pour les enseignants qui mettent en avant le plaisir d'enseigner, l'aspect relationnel du métier est premier». Dans une recherche centrée sur les émotions en classe, Cowie (2011) a également montré le poids de la relation à l'élève sur les émotions des enseignants. D'ailleurs, il est intéressant de noter que les élèves évoquent aussi la relation à l'enseignant comme l'inducteur émotionnel positif principal, d'après une recherche prenant appui sur l'EPS (Petiot, Desbiens \& Visioli, 2014). Lorsque Catherine ressent en danse un plaisir estimé à +4 , elle insiste sur l'ambiance de classe, à la fois studieuse et propice à l'humour. Notre recherche rejoint ainsi les réflexions de Durand (2001) qui avait déjà pointé l'importance du rire en classe, en soulignant que certains professeurs d'EPS cultivaient un talent d'humoriste, principalement avec l'intention d'imprimer une tonalité, d'établir une ambiance qui soit à la fois studieuse et détendue. Comme l'énoncent Petitjean et Proego-Valverde (2013), il est indéniable qu'enseignant et élèves, à l'image de la situation vécue par Catherine, participent conjointement de l'humour en classe, par la confrontation de leurs réactions respectives. Dans une perspective philosophique, Dumouchel (1999) considère que le plaisir, et plus généralement les émotions, doivent être compris comme inclus dans un processus de coordination émotionnelle, c'est à dire de contagion des émotions entre les différents acteurs. La disponibilité de l'enseignant s'illustre alors dans sa capacité à capter les indices de plaisir chez les élèves (Bui-Xuan \& Haye, 2007). 


\section{eJRIEPS 40 janvier 2017}

Malgré tout, les enseignants experts sont plusieurs à estimer à certains moments de la leçon des valeurs plus négatives et plus intenses sur l'EPD. C'est le cas de Paul qui au début du cours de danse se trouve face à des élèves qui n'écoutent pas les consignes. Les émotions des enseignants sont principalement liées aux comportements des élèves jugés inappropriés. Concernant Catherine, c'est tout au long du cours qu'elle ressent un manque de réaction de ses élèves en badminton, sans parvenir selon elle à les faire bouger. Ce résultat met en avant le processus de contagion des émotions négatives en classe, également mis en évidence par Bakker et Schaufeli (2000) dans une recherche sur le burn out dans le domaine scolaire. Comme l'explique Dumouchel (1999), les émotions constituent « des actions et des stratégies dont les agents sont responsables, sans que, pour autant, elles soient entièrement en leur pouvoir» (p. 17). Par les processus de coordination émotionnelle, l'enseignant peut chercher à influencer ses émotions et celles des élèves en classe. Toutefois, ces processus sont davantage le fruit du couplage enseignant-environnement que le résultat de stratégies prédéterminées, y compris pour des experts.

Plus récemment, Chen (2016) a réalisé un inventaire des sources des émotions positives (joie, amour) et négatives (tristesse, colère, peur) d'enseignants chinois de primaire, montrant que ces derniers perçoivent positivement la relation aux élèves et aux collègues mais ressentent des émotions négatives liées notamment à la compétition perçue entre collègues, à l'ambivalence de la vie au travail et à la pression sociale. Notre étude offre un éclairage plus microscopique de l'analyse des émotions des enseignants en analysant ces dernières en situation et sans sélectionner d'émotions a priori. Elle met également particulièrement l'accent sur leur caractère fondamentalement social. A ce titre, dans le domaine scolaire, Visioli, Petiot et Ria (2015) notent un glissement des recherches liées aux émotions en classe vers une conception plus sociale, c'est-à-dire vers l'étude de dynamiques émotionnelles collectives, incluant les dimensions corporelles et les questions d'expressivité.

5. 2. La dynamique émotionnelle des enseignants d'EPS : quelles différences entre les experts et les novices ?

Les résultats obtenus au cours de cette étude ont permis de montrer que l'intensité et la valence des émotions des enseignants experts contrastent avec celles ressenties par les enseignants débutants, caractérisées par des variations importantes et des valeurs souvent extrêmes et négatives sur l'échelle (Ria et al., 2004). L'activité des enseignants débutants se caractérise notamment par une difficulté à anticiper sur ce qui va se jouer 


\section{eJRIEPS 40 janvier 2017}

concrètement en classe, et cette indétermination est vécue avec anxiété. Par contre, nos résultats montrent que le cours d'EPS est devenu un « micro-monde » familier pour les enseignants experts (Varela, 1996), c'est à dire un ensemble de situations typiques construites sur la base de significations et d'émotions récurrentes, au sein desquelles l'acteur mobilise des savoir-faire implicites, des dispositions à agir la plupart du temps transparentes. Ils sont rarement surpris par les interactions en classe, ce qui explique pour partie leur dynamique émotionnelle stable, modérée et plutôt positive. Dès lors, le spectre des émotions ressenties par l'enseignant peut constituer un bon indicateur du niveau de développement de l'activité professionnelle (Visioli \& Ria, 2010).

Plus précisément, nos résultats soulignent le lien entre cette dynamique émotionnelle et leurs connaissances mobilisées en situation de classe. Construites au fil de l'expérience professionnelle (Rohaan, Taconis \& Jochems, 2010), elles leur confèrent des attentes claires et une conviction dans l'interaction avec les élèves, contrairement aux novices qui ont davantage de difficultés à situer le niveau d'exigence à instaurer (Ria, 2014). En particulier, les experts mobilisent des connaissances liées aux élèves, notamment à leur rapport à l'APSA. En témoigne par exemple les propos de Philippe au sujet des difficultés de Melissa, notamment concernant l'anticipation coïncidence en badminton, dans la continuité des recherches analysant la manière dont les enseignants prennent en compte l'activité d'élèves particuliers, en grande difficulté ou, à l'inverse, spécialistes de l'APSA (Crance, Trohel \& Saury, 2013). Plus généralement, les experts mobilisent des connaissances sur la personnalité des élèves, ce qui leur permet d'anticiper certains évènements inattendus susceptibles de se produire en classe. Christophe, qui a typicalisé les réactions potentiellement épidermiques de Yunus, s'attend ainsi à certains comportements inappropriés de sa part. Ceci rappelle l'intérêt de connaître et de prendre en compte l'état émotionnel des élèves en EPS. A ce titre, Mc Caughtry et Rovegno (2003) ont analysé le développement des connaissances pédagogiques d'enseignants d'EPS novices durant 20 leçons de volley-ball, montrant la construction progressive de connaissances liées à la prise en compte des émotions manifestées par les élèves.

Finalement, nos résultats rappellent qu'une des particularités de l'activité des enseignants réside dans la prise en compte des émotions des élèves pour favoriser une dynamique collective positive, alors qu'ils sont eux-mêmes en situation d'en ressentir (Ria, 2006). A ce titre, les préoccupations des enseignants experts témoignent de l'empathie développée vis-à-vis des élèves, qui repose sur une aptitude sensorielle à percevoir les indices et les signaux émis par le corps de l'autre (Cyrulnik, 2007). Plus précisément, elle relève 


\section{eJRIEPS 40 janvier 2017}

prioritairement d'une capacité à résoudre un problème spatial consistant à naviguer entre plusieurs référentiels. Celui qui a le « sens de l'autre » sait jongler entre plusieurs stratégies d'orientation dont la stratégie de "route " (égocentrée et en première personne) et la stratégie de « survol » (allocentréee et en troisième personne) (Berthoz, 2004). C'est en cela que les enseignants experts perçoivent directement l'état émotionnel des élèves, notamment à partir des formes de corps des élèves, à l'image de François concernant l'élève en retard. Ils ne se montrent pas pour autant sympathiques (Jorland, 2004), c'est-à-dire qu'ils ne se risquent pas à ressentir les mêmes émotions que les élèves, comme le font plus souvent les enseignants novices.

5. 3. Entre sentiment de contrôle et prise de distance des enseignants : quels

processus de régulation émotionnelle en situation?

Les résultats obtenus à travers cette recherche montrent que les enseignants oscillent entre la préservation d'un sentiment de contrôle et une prise de distance permettant la régulation de leurs émotions en situation. Ces processus concourent à expliquer leur dynamique émotionnelle stable, modérée et positive lors des leçons.

Premièrement, il semble que les enseignants cherchent à préserver leur sentiment de contrôle lors de la leçon, de plusieurs manières. D'une part, comme le souligne Foucault (1975), l'organisation de l'espace, du temps et des rituels sont des médiateurs du pouvoir permettant de discipliner, c'est-à-dire de contrôler. A ce titre, à l'instar de Catherine en Danse, l'autorité passe par l'imposition de présenter la chorégraphie malgré les réticences des élèves. Or, Bandura (2003) pose que le sentiment d'être efficace à contrôler des événements joue un rôle central dans l'activation des émotions. Plus un enseignant croit être en mesure de pouvoir contrôler les situations difficiles en classe, moins il s'inquiète (Gaudreau, Royer, Beaumont, Frenette, 2012). C'est le cas de François en Badminton, qui manifeste des émotions positives liées à un sentiment de contrôle lui permettant de « voir loin », d'anticiper sur les comportements des élèves en tant que spécialiste de l'APSA.

Deuxièmement, il arrive durant les leçons que les enseignants soient confrontés à une relative perte de contrôle. Dans ce cas, ils semblent faire preuve d'une prise de recul leur permettant de maintenir une dynamique émotionnelle stable et modérée, à l'image de Paul en danse. Ces résultats marquent une distinction entre l'activité des experts et celle des novices qui, en cas de perte de contrôle, se laissent souvent aller à des émotions très négatives. Cette perte de contrôle passe chez eux fréquemment par l'apparition de dilemmes (Ria et al., 2004), ouvrant à des conflits intrapsychiques (Bertone, Méard, Euzet, Ria \& Durand, 2003) et à des cercles vicieux dont il est difficile de s'échapper (Flavier, 


\section{eJRIEPS 40 janvier 2017}

Bertone, Hauw \& Durand, 2002). Si les experts vivent eux aussi un certain nombre de situations problématiques, génératrices de dilemmes, ils ressentent des émotions d'intensité plus modérée grâce à une prise de distance permettant de faire face à ces effets circulaires. Dans le cas de Paul, nous constatons que l'humour est un moyen d'y parvenir. Finalement, cette prise de distance semble témoigner, à l'instar de Watzlawick (1988), d'une capacité de voir les choses autrement, sous un autre angle, de dépasser la lecture actuelle que l'on fait d'une situation pour lui en substituer une autre.

Le phénomène de régulation des émotions est défini dans un cadre cognitiviste comme « le processus par lequel les individus influencent quelles émotions ils ont, quand ils les ont, et comment ils ressentent et expriment ces émotions »(Gross, 1998, p. 275). Plus précisément, trois types de stratégies de régulation des émotions sont généralement distinguées dans cette perspective théorique : (1) les stratégies de ré-évaluation cognitive de la situation ; (2) les stratégies de suppression des émotions ; (3) les stratégies basées sur l'acceptation des émotions (Gross, 2011). Néanmoins, il nous semble difficile de réduire la prise de distance de Catherine uniquement à l'idée d'une régulation maitrisée, autonome et interne, le contexte de classe et l'activité des élèves étant indissociables de ses émotions ressenties. Finalement, notre étude permet de décrire ce phénomène de régulation des émotions chez les enseignants d'EPS experts en apportant une analyse plus dé-psychologisante tendant à inscrire leurs émotions dans l'activité située sans réduire cette régulation, à l'instar de Leroy, Delelis, Nandrino et Chistophe (2014), à un phénomène uniquement endogène. Par ailleurs, si les propos des enseignants renseignent cette prise de recul participant d'une dynamique émotionnelle stable, modérée et plutôt positive en cours, ils expliquent difficilement les processus y participant. Korb (2009) affirme que les processus de régulation émotionnelle peuvent être à la fois conscients et inconscients, automatiques ou contrôlés. Selon lui, il existerait un continuum allant des phénomènes de régulation émotionnelle automatique à ceux qui sont plus de l'ordre du conscient et du volontaire.

5. 4. Quelles conséquences sur la formation initiale et continue des enseignants ?

Pour finir, les résultats obtenus à travers cette étude présentent des intérêts dans le cadre de la formation initiale, pour enseigner aux novices à stabiliser leurs émotions ressenties, et continue des enseignants d'EPS, pour contribuer au plaisir d'enseigner et à la lutte contre d'éventuelles routines. Plus largement, les intérêts de cette recherche se situent dans le développement d'une "sensibilité à », définie dans l'approche phénoménologique (Récopé, Rix-Lièvre, Fache, Boyer, 2013) comme organisatrice de 


\section{eJRIEPS 40 janvier 2017}

l'expérience des acteurs engagés dans la situation. La disponibilité est comprise dans notre étude comme «disponibilité à », ce qui participe à actualiser concrètement et pratiquement un ensemble complexe de normes exprimant des orientations privilégiées portant l'individu vers autrui.

Premièrement, en ce qui concerne la formation initiale, Ria (2009) souligne que l'activité débutante analysée et interprétée à l'aune de celle des experts conduit davantage à une caractérisation en creux de ce que le débutant n'est pas, qu'à une réelle investigation de ce qu'il est, ce qu'il ressent et interprète en situation professionnelle. Dès lors, à l'instar du positionnement de Leblanc, Ria, Dieumegard, Serres \& Durand (2008), nous sommes d'avis que ne pas prendre en compte ces différences de signification risque de conduire à définir des objectifs de formation pour les novices inatteignables à ce moment-là de leur trajectoire professionnelle, et non pertinents par rapport à leurs mondes propres. Si ces réserves sont justifiées, elles ne peuvent selon nous masquer l'intérêt d'également faire référence à l'expertise. II s'agit en effet de reconnaître une sagesse de la pratique et de l'activité réelle, d'admettre que les acteurs savent beaucoup de choses, que leurs savoirs sont nichés dans l'action (Durand, De Saint-Georges \& Meuwly-Bonte, 2006). Selon ces auteurs, il s'agit de conceptualiser des dispositifs de formation comme des « espaces d'actions encouragées », c'est à dire des précurseurs d'actions et d'expériences supposées induire un développement et un apprentissage, ce qui nécessite de la part des formateurs une bonne connaissance des étapes du développement de l'activité professionnelle, et donc de l'expertise en enseignement.

Finalement, en accord avec les tenants d'une anthropologie culturaliste, notre recherche peut s'imposer comme le support d'un « enseignement ostensif » (Wittgenstein, 1996) des règles, par lequel les formateurs fondent la signification de faits et d'actions professionnels considérés comme exemplaires (Bertone, 2011). En d'autres termes, dans cette perspective, l'interprétation correcte par les apprenants des expériences professionnelles vécues en classe suppose un apprentissage préalable, qui fait ensuite l'objet d'« explications » (Wittgenstein, 1996), et éventuellement de débats critiques entre l'enseignant et l'apprenant. Ce n'est que par la suite, au travers de la pratique conforme des règles dans les différents contextes, que les apprenants parviennent à suivre intentionnellement les règles apprises. Sans être prescriptive, l'analyse rapportée de l'activité d'enseignants experts, avec une focale sur leurs émotions ressenties en classe, peut donc figurer comme un « exemple exemplaire » permettant d'illustrer, sous la forme d'un apprentissage ostensif des règles, comment maintenir une dynamique émotionnelle 


\section{eJRIEPS 40 janvier 2017}

stable et modérée, notamment pour pouvoir se montrer plus disponible aux élèves (Bertone, 2011).

Deuxièmement, dans le cadre des formations continues d'enseignants, cette étude pose la question de la prise en compte des émotions, et notamment du plaisir d'enseigner (Carlier, 2011). L'enjeu pourrait être de questionner la manière de créer, ponctuellement, des perturbations de cette dynamique pour amener des émotions plus intenses et positives. Les moments à plus forte tonalité émotionnelle sont en effet susceptibles de jouer un rôle dans la construction, par les enseignants, de nouvelles connaissances (Terré, 2013). Plus encore, oser s'engager dans des situations plus incertaines, improviser, ou encore jouer avec ses émotions en classe (Visioli et al., 2014), sont autant de pistes susceptibles d'aider les experts à lutter contre les éventuelles routines inhérentes au métier d'enseignant. Par exemple, nos résultats montrent que les enseignants experts ressentent parfois des émotions très positives lorsqu'ils enseignent une APSA pour laquelle ils ne sont pas spécialistes, comme c'est le cas de François qui improvise la résolution collective d'un problème en arts du cirque. Plus généralement, la plupart des émotions très positives survenues ont lieu lors d'APSA pour lesquelles les enseignants se sont déclarés non spécialistes (danse et arts du cirque). Lors de ces leçons, les experts mobilisent des connaissances transversales qui leur permettent de compenser pour partie un manque de spécialisation dans l'APSA enseignée et de faire preuve d'une certaine créativité face aux élèves (Sawyer, 2004), ressentant ainsi des émotions très positives.

\section{Conclusion}

Au-delà des éléments discutés précédemment, notre étude pose de manière plus générale la réflexion des relations entre connaissances et émotions dans l'activité des enseignants et rappelle l'importance d'une formation par l'action pour le développement de connaissances contextualisées (Durand et Arzel, 2002). Dans notre étude, les connaissances mobilisées par les experts ont une importance majeure dans leur dynamique émotionnelle stable et modérée, sont intimement liées aux élèves et indissociables de la situation dans laquelle elles émergent. A ce titre, Méard, Flavier et Chaliès (2008) proposent un plaidoyer pour une EPS comprise et défendue à partir de l'analyse de l'activité réelle, afin de se dégager d'une perception virtuelle de la discipline. Notre étude va plutôt dans le sens d'une indissociabilité des connaissances et des émotions, qui, plutôt que d'être entendues dans une relation concurrentielle, devraient être 


\section{eJRIEPS 40 janvier 2017}

considérées comme articulées. D’ailleurs, Ubaldi (2006) affirme que sa carrière d'enseignant lui a appris « que le relationnel était incontournable, mais qu'une démarche rigoureuse s'imposait, car, sans cadre d'action cohérent, on n'existe pas. Paradoxalement, la centration sur les savoirs va donner du sens à l'émotion, à la relation, à l'affectif. Didactique, pédagogie, relation, émotion, tout s'imbrique et ne s'exclut plus » (p. 230).

\section{Bibliographie}

Andrieu, B. (2003). Corps, peau, silences, dans l'enseignement. Nancy : CRDP Lorraine.

Bakker, A. B., \& Schaufeli, W. B. (2000). Burnout contagion processes among teachers. Journal of Applied Social Psychology, 30, 1-21.

Bandura, A. (2003). Auto-efficacité. Le sentiment d'efficacité personnelle. Paris: De Boeck.

Barbier, J.M. \& Galatanu. O. (1998). Action, affects et transformation de soi. Paris : PUF.

Berthoz, A. (2004). La physiologie du changement de point de vue. In A. Berthoz et G. Jorland (dirs.), L'empathie (pp. 156-167). Paris, Odile Jacob, 2004.

Bertone, S. (2011). La force des règles dans l'apprentissage du métier d'enseignant en formation par alternance. Note de synthèse pour l'Habilitation à Diriger des Recherches. Université de la Réunion.

Bertone, S., Méard, J.A., Euzet, J.-P., Ria, L. \& Durand, M. (2003). Intrapsychic conflict experienced by a preservice teacher during classroom interactions : A case study in physical education, Teaching and Teacher Education, 19(1), 113-125.

Bui-Buan, G. (2011). Le plaisir, un fait conatif total. In G. Haye (dir.), Le plaisir (pp. 49-63), Paris, Editions Revue EPS.

Bui-Xuan, G. \& Haye, G. (2007). La sensibilité des enseignants d'EPS au plaisir des élèves. Enquête de l'AEEPS 2005-2007. In P. Gagnaire et F. Lavie (Eds.), Le plaisir des élèves en éducation physique et sportive : futilité ou nécessité ? (129144). Montpellier : Coédition AEEPS - AFRAPS.

Carlier, G. (2011). Plaisir d'enseigner : la quête du Graal ? In G. Haye (dir.), Le plaisir (pp. 99-117), Paris : Editions Revue EPS.

Chen, J. (2016). Understanding teacher emotions: The development of a teacher emotion inventory, Teaching and Teacher Education, 55, 68-77.

Colombetti, G. \& Thompson, E. (2008). The feeling body: towards an enactive approach to emotion. In Overton WF, Muller U, Newman JL (eds) Developmental Perspectives 


\section{eJRIEPS 40 janvier 2017}

on Embodiment and Consciousness (pp. 45-68), New York: Lawrence Erlbaum Ass.

Cowie, N. (2011). Emotions that experienced English as a Foreign Language (EFL) teachers feel about their students, their colleagues and their work, Teaching and Teacher Education, 27(1), 235-242.

Crance, M-C., Trohel, J. \& Saury, J. (2013).The experience of highly skilled student during handball lessons in physical education : a relevant pointer to the gap between school and sports contexts of practice. Physical education and sport pedagogy, 18(1), 103-115.

Cyrulnik, B. \& Pourtois, J-P. (2007). Ecole et Résilience. Paris : Odile Jacob.

Damasio, A.R. (1999). Le sentiment même de soi : corps, émotions, conscience. Paris : Editions Odile Jacob.

Dicke, T., Parker, P. D., Holzberger, D., Kunina-Habenicht, O., Kunter, M. \& Leutner, D. (2015). Beginning teachers' efficacy and emotional exhaustion: Latent changes, reciprocity, and the influence of professional knowledge, Contemporary Educational Psychology, 41, 62-72.

Dumouchel, P. (1999). Emotions : essai sur le corps et le social. Le Plessis-Robinson : Institut Synthélabo.

Durand M. (2001). Chronomètre et survêtement : Reflets de l'expérience quotidienne d'enseignants d'éducation physique. Paris : Editions Revue EPS.

Durand, M., Arzel, G. (2002). Autonomie et connaissance dans les conceptions de l'apprentissage, de l'enseignement et de la formation des maîtres. In M. Carbonneau et M. Tardif (Eds.), Les réformes en éducation, leurs impacts sur l'école et sur la formation des maîtres (pp. 61-77). Sherbrooke : Edition du CRP.

Durand, M., De Saint-Georges, I. \& Meuwly-Bonte, M. (2006). Le curriculum en formation des adultes : argumentation pour une approche orientée activité. Raisons d'Agir, 10, 57-76.

Flavier, E., Bertone, S., Hauw, D. \& Durand, M. (2002). The meaning and organization of physical education teachers' action during conflict with students. Journal of Teaching in Physical Education, 22, 20-38.

Foucault, M. (1975). Surveiller et punir. Paris : Gallimard.

Gaudreau, N., Royer, E., Beaumont, C., Frenette, E. (2012). Le sentiment d'efficacité personnelle des enseignants et leurs pratiques de gestion de la classe et des 


\section{eJRIEPS 40 janvier 2017}

comportements difficiles des élèves, Revue Canadienne de l'Education, 35(1), 82101.

Gross, J. J. (1998). The emergine field of emotion regulation : an integrative review, Review of general psychology, 2(3), 271.

Korb, S. (2009). La régulation des émotions. In D. Sander \& K. Scherer, Traité de psychologie des émotions (pp. 259-288), Paris : Dunod.

Lave, J. (1988). Cognition in practice. Mind, mathematics and culture in everyday life. Cambridge : Cambridge University Press.

Lazarus, R.S. (2001). Relationnal meaning and discrete emotions. In K.S. Sherer, A. Schorr et T. Johnstone (Eds.), Appraisal processes in emotion: theory, methods, research (pp. 56-68). New York: Oxford University Press.

Leblanc, S., Ria, L., Dieumegard, G., Serres, G., \& Durand, M. (2008). Concevoir des dispositifs de formation professionnelle des enseignants à partir de l'analyse de l'activité dans une approche enactive, @ctivités, 5(1), 58-78.

Leroy, T., Delelis, G., Nandrino, J-L. \&Chistophe, V. (2014). Régulations endogène et exogène des émotions: des processus complémentaires et indissociables, Psychology française, 59, 183-197.

Masciotra, D., Roth, W.M. \& Morel, D. (2008). Enaction. Apprendre et enseigner en situation. Bruxelles : Editions de Boeck.

Mattern, J. \& Bauer, J. (2014). Does teachers' cognitive self-regulation increase their occupational well-being? The structure and role of self-regulation in the teaching context, Teaching and Teacher Education, 43, 58-68.

McCaughtry, N. \& Rovegno, I. (2003). Development of pedagogical content knowledge: moving from blaming students to predicting skilfulness, recognizing motor development, and understanding emotion, Journal of Teaching in Physical education, 22, 355-368.

Méard, J., Flavier, E. \& Chaliès, S. (2008) Comprendre et défendre l'EPS par l'analyse de l'activité des enseignants et des élèves, Revue Hyper, 241, 25-28.

Mikolajczak M. \& Bausseron E. (2013). Les compétences émotionnelles chez l'adulte. In O. Luminet (dir), Psychologie des émotions. Nouvelles perspectives pour la cognition, la personnalité et la santé (pp. 129-173). Bruxelles: De Boeck supérieur.

Obin (2002). Enseigner, un métier pour demain. Rapport au ministre de l'Education Nationale. 


\section{eJRIEPS 40 janvier 2017}

Petiot, O., Desbiens, J-F., et Visioli, J. (2014). Perceptions d'élèves du secondaire concernant leurs inducteurs émotionnels en EPS, eJRIEPS, 32, 4-37.

Petitjean, C., \& Priego-Valverde, B. (2013). Pourquoi et comment faire de l'humour en classe ? Les représentations sociales de la compétence humoristique dans des interactions didactiques en Suisse romande, Langage et Société, 144, 41-63.

Philippot, P. \& Feldman, R. S. (2004). The regulation of emotion. Mahwah, NJ : Erlbaum.

Rascle N., Bergugnat-Janot L. \& Hue S. (2011). Travail émotionnel et burnout : état de la question et actions de prévention, l'exemple des enseignants. In L. Lafortune, P.

A. Doudin, D. Curchot- Ruedi \& N. Lafranchise, La santé des enseignants (pp. 137-155), Presses de l'Université du Québec.

Récopé, M., Rix-Lièvre, G., Fache, H. \& Boyer, S. (2013). La sensibilité à, organisatrice de l'expérience vécue, in L. Albarello, J-M. Barbier, E. Bourgeois \& M. Durand (eds), Expérience, Activité, Apprentissage (pp. 111-133). Paris : PUF.

Ria, L. (2006). L'entrée dans le métier des enseignants du second degré : un programme de recherche centré sur l'analyse de l'activité. Note de synthèse en vue de l'obtention à l'habilitation à diriger des recherches, Université de Clermont Ferrand.

Ria, L. (2009). De l'analyse de l'activité des enseignants débutants en milieu difficile à la conception de dispositifs de formation. In M. Durand \& L. Filliettaz (Eds.), La place du travail dans la formation des adultes. Paris : PUF.

Ria, L., Sève, C. Durand, M. \& Bertone, B. (2004). Indétermination, contradiction et exploration : Trois expériences typiques des enseignants débutants en Education Physique. Revue des Sciences de l'Education, 3(3), 535-554.

Ric, F., \& Alexopoulos, T. (2009). Etats affectifs et traitement de l'information sociale. Revue Electronique de Psychologie Sociale, 4, 40-46.

Rohaan, E. J., Taconis, R., \& Jochems, W. M. G. (2010). Reviewing the relations between teachers' knowledge and pupils' attitude in the field of primary technology education. International Journal of Technology and Design Education, 20(1), 1526.

Rosch, E. (1978). Principles of categorization. In E. Roschet B.B. Llyod (Eds.), Cognition and categorization (27-48). Hillsdale : Lauwrence Erlbaum Associates.

Sawyer, R.K. (2004). Creative Teaching: Collaborative Discussion as Disciplined Improvisation, Educational Researcher, 33(2), 12-20.

Sève, C., Theureau, J., Saury, J. \& Haradji, Y. (2012). Drôle d'endroits pour une rencontre 


\section{eJRIEPS 40 janvier 2017}

: STAPS, ergonomie et cours d'action. In M. Quidu (Ed.), Les Sciences du sport en mouvement : Innovations et traditions théoriques en STAPS (pp. 39-64). Paris : L'Harmattan.

Stojiljkovic, S., Stojanovic, A. \& Doskovic, Z. (2011). Moral reasoning and empathy in Serbian teachers. Oral presentation submitted on International Conference on Education and Educational Psychology ICEEPSY 2011, Istanbul, Turkey, Abstracts, 133-134.

Sutton, R.E. (2004). Emotional regulation goals and strategies of teachers. Social Psychology of Education, 7(4), 379-388.

Terré, N., Saury, J., \& Sève, C. (2013). Emotions et transformation des connaissances en éducation physique : une étude de cas en kayak de mer, eJRIEPS, 29, 27-58.

Theureau, J. (2004). Le cours d'action : méthode élémentaire. Toulouse: Octarès

Theureau, J. (2006). Le cours d'action : Méthode développée. Toulouse : Octarès.

Tochon, F. (1993). L'enseignant expert. Paris : Nathan.

Ubaldi, J.L. (2006). L'EPS dans les classes difficiles. Paris : Revue EPS.

Varela, F. (1999). Quatre phares pour l'avenir des sciences cognitives. Théorie Littérature Enseignement, 17, 7-21.

Varela, F.J. (1989). Autonomie et connaissance. Essai sur le vivant. Paris : Seuil.

Varela, F.J. (1996). Quel savoir pour l'éthique ? Action, sagesse et cognition. Paris : La Découverte.

Visioli, J. \& Ria, L. (2010). L'expertise des enseignants d'EPS : Quelle prise en compte du contexte et des émotions ? Science et Motricité, 71, 3-19.

Visioli, V. Petiot, O. \& Ria, L. (2014). Le jeu émotionnel des professeurs experts en cours d'éducation physique et sportive : un moyen de favoriser le plaisir d'enseigner?, Science et Motricité, publié en ligne : DOI: 10.1051/sm/2014008.

Visioli, V. Petiot, O. \& Ria, L. (2015). Vers une conception sociale des émotions des enseignants ?, Carrefours de l'éducation, 40, 201-230.

Watzlawick, P. (1988). L'invention de la réalité: contributions au constructivisme. Paris : Seuil.

Wittgenstein, L. (1996). Recherches philosophiques. Paris : Gallimard. 